\title{
Design of the Congestion Control for TCP/AQM Network with Time-Delay
}

\author{
Dazhong Wang and Shujing Wu \\ Shanghai University of Engineering Science, Shanghai 201620, China \\ Correspondence should be addressed to Dazhong Wang; wdzh168@hotmail.com
}

Received 8 July 2014; Accepted 20 August 2014; Published 25 September 2014

Academic Editor: Housheng Su

Copyright (C) 2014 D. Wang and S. Wu. This is an open access article distributed under the Creative Commons Attribution License, which permits unrestricted use, distribution, and reproduction in any medium, provided the original work is properly cited.

\begin{abstract}
The purpose of this paper is to design congestion controller for TCP/AQM (transmission control protocol/active queue management) networks using model following control; the equilibrium of a class of TCP/AQM networks with time-delay is investigated, and the effect of communication time-delay on the stability is addressed. The features of this design method are bounded property of the internal states of the control system being given and the utility of this control. Such design exhibits important attributes including fast convergence with high accuracy to a desired queue length. Simulation results show that the time-delay nonlinear behavior of the system can be controlled by this method.
\end{abstract}

\section{Introduction}

In recent years, with the rapid growth of throughput-demanding applications, congestion control has emerged as a major issue in computer and communication network design [1]. So many researchers are seeking some methods to effectively control congestion. TCP congestion control mechanism is used to prevent congestion collapse.

AQM schemes have been proposed to complement the TCP network congestion control [2]. Several mathematical models are developed by some researchers [3-5] and a variety of control theory-based AQM schemes are proposed based on these models. The simulated approaches contain a wide range of variations in network topologies, topological parameters, load and capacity, and traffic mixtures. The outperformance of the PFC-AQM in comparison with the commonest AQM methods such as the RED (random early detection), PI, and REM (random early marking) emphasizes the proper applicability of PFC as an AQM method [69]. Based on the system model for congestion control in transmission control protocol TCP/AQM networks, control theory-based approaches are utilized either to analyze or to design the AQM schemes. Based on the system model, several conventional controllers [10-15] are designed as AQM methods in TCP networks.
The design of some communication systems requires the implementation of time-delays within the system. These time-delays can be accomplished with a variety of optics technologies, which could be readily fabricated and integrated into the communication system without significant impacts on the system design [16-19]. Time-delay is very important for the modeling of networks, occurring both in the control of networks and in the control over networks [20]. In the context of communication networks, the term "congestion control" is generally used to refer to the action of regulating various flows within a network. In recent years, intense research efforts are devoted to the application of the Smith predictor for queue length control of ATM (asynchronous transfer mode) networks [21]. The TCP was designed in the late 1983s by Jacobson, which is a critical part of the internet machinery.

The purpose of this paper is to design a congestion controller based on the model following control system (MFCS) $[22,23]$ control theory. The features of this design method are that bounded property of the internal state of the system, which is given and confirmed on basis of a numerical example of the network congestion system in which the output signal of the control system asymptotically follows the reference model signal in the case of the existence of disturbances. 
The paper is organized as follows. In Section 2, the TCP/AQM network in congestion control model is described. In Section 3, controller design of the network congestion system with time-delay is proposed. In Section 4, bounded analysis of control system internal state is shown. Section 5 is the simulation results. The paper is concluded in Section 6.

\section{The TCP/AQM Networks in Congestion Control Model}

In this paper, the network in Figure 1 is considered. The network consists of $n$ nodes (sender), 1 node (receiver), and 1 bottleneck router. The bottleneck router sends packets from these senders to the receiver. This network topology denotes 1 server machine to multiple client machines in a computer network. TCP is only the communication protocol in Figure 1. Large-scale networks can be simplified as in Figure 1 in case of designing congestion controllers if only one router is bottleneck in the large-scale computer network.

In this approach, we overview the dynamical fluid-flow model developed by [24-29] to describe the behavior of TCP/AQM networks. A simplified version of that system model is considered, which ignores the timeout and slow start mechanism of TCP. The model involves the average value of key network variables and is described by the following coupled nonlinear differential equations with time-delay [25]:

$$
\begin{gathered}
\dot{W}(t)=\frac{1}{R(t)}-\frac{W(t)}{2} \frac{W(t-R(t))}{R(t-R(t))} p(t-R(t)), \\
\dot{q}(t)=\frac{W(t)}{R(t)} N(t)-D(t), \\
R(t)=\frac{q(t)}{D(t)}+T_{p},
\end{gathered}
$$

where $W(t)$ is the congestion window size in packets at time $t, q(t)$ is the queue length at the congested router in packets, $R(t)$ is the RTT (round trip time) which represents the timedelay in TCP dynamics in seconds, $D(t)$ is the link capacity in packets per second, $T_{p}$ is the propagation time-delay in seconds, $N(t)$ is the number of active TCP connections, and $p(t)$ is the packet mark/drop probability.

These differential equations in the block diagram of Figure 2 are taken from [24] highlighting TCP windowcontrol and queue dynamics.

Set up a model for nonlinear TCP networks dynamic model. Let $x_{1}(t)=q(t)-q_{0}, x_{2}(t)=\dot{x}_{1}(t)$, and $u(t)=p(t)$, where $q_{0}$ is a desired queue length in the router. Assume that the rate of the change for $x_{1}(t), N(t), C(t)$, and $R(t)$ is slower than $W(t), q(t)$; (1) can be expressed in the following form [30]:

$$
\dot{x}(t)=f(x(t))+g(x(t)) u(t-R(t)),
$$

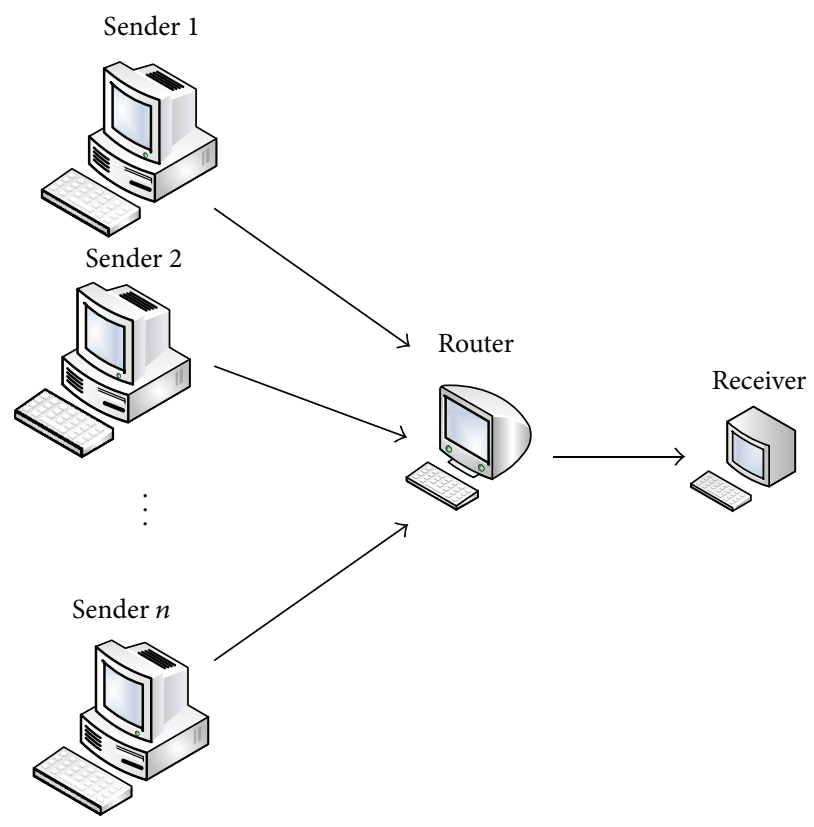

FiguRE 1: The network of the congestion control.

where

$$
\begin{aligned}
x(t) & =\left[\begin{array}{ll}
x_{1}(t) & x_{2}(t)
\end{array}\right]^{T}, \\
f(x(t)) & =\left[\begin{array}{ll}
f_{1}(x(t)) & f_{2}(x(t))
\end{array}\right]^{T}, \\
g(x(t)) & =\left[\begin{array}{ll}
g_{1}(x(t)) & g_{2}(x(t))
\end{array}\right]^{T}, \\
f_{1}(x(t)) & =x_{2}(t) \\
f_{2}(x(t)) & =\frac{N(t)}{R^{2}(t)}, \\
g_{1}(x(t)) & =-\frac{\left[\left(x_{2}(t)+C(t)\right]+\left[x_{2}(t-R(t))+C(t)\right]\right.}{2 N(t)} .
\end{aligned}
$$

Therefore, the system is defined by

$$
\begin{gathered}
\dot{x}(t)=f(x(t), u(t-R(t))), \\
y(t)=\left[\begin{array}{ll}
0 & 1
\end{array}\right] x(t) .
\end{gathered}
$$

\section{Controller Design of the Network Congestion System with Time-Delay}

Based on (6), the system in (4) can be rewritten in equivalent form as follows:

$$
\begin{gathered}
\dot{x}(t)=A_{11} x(t)+A_{12} u(t)+f_{1}(x(t), u(t-R(t))), \\
\dot{u}(t)=A_{21} x(t)+A_{22} u(t)+g_{1}(x(t), u(t))+B u_{1}(t),
\end{gathered}
$$

where $A_{11} \in R^{n \times n}, A_{12} \in R^{n \times l}, A_{21} \in R^{l \times n}$, and $A_{22} \in R^{l \times l}$. 


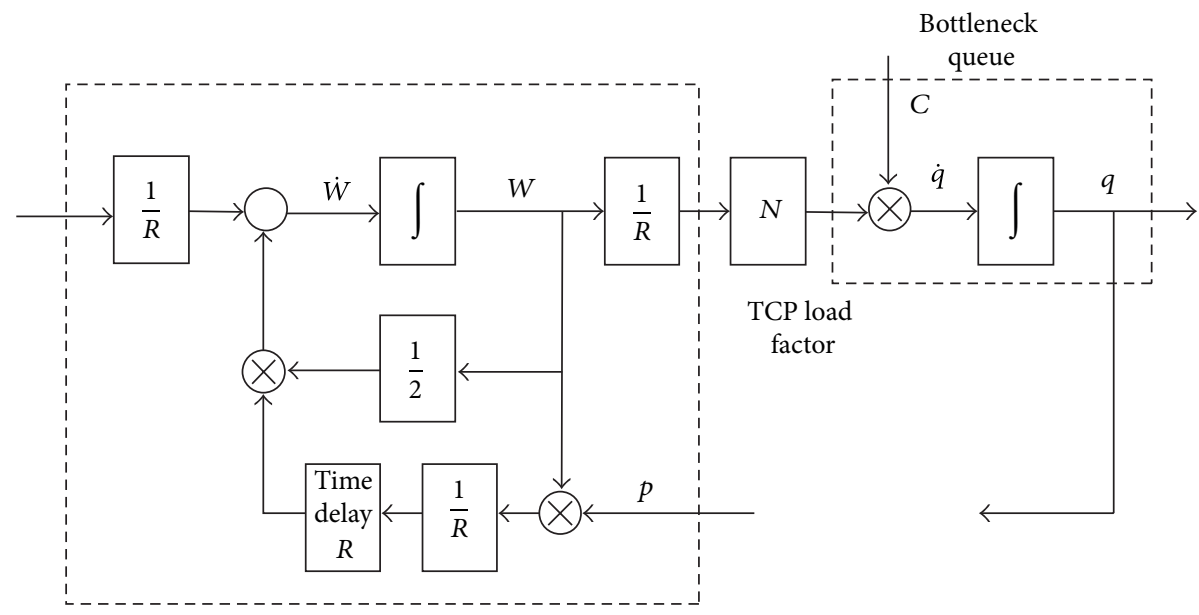

FIgURE 2: Block diagram of TCP congestion avoidance mode.

Then, from (8), we can obtain the following system:

$$
\begin{gathered}
\dot{x}_{1}(t)=A_{1} x_{1}(t)+B_{1} u_{1}(t)+f_{2}\left(x_{1}(t)\right), \\
y(t)=C_{1} x_{1}(t),
\end{gathered}
$$

where

$$
\begin{aligned}
x_{1}(t) & =\left[\begin{array}{ll}
x(t) & u(t)
\end{array}\right]^{T}, \\
f_{2}\left(x_{1}(t)\right) & =\left[\begin{array}{ll}
f_{1}\left(x_{1}(t)\right) & g_{1}\left(x_{1}(t)\right)
\end{array}\right]^{T}, \\
f_{1}\left(x_{1}(t)\right) & =f\left(x_{1}(t)\right)-A_{11} x(t)-A_{12} u(t), \\
A_{1} & =\left[\begin{array}{ll}
A_{11} & A_{12} \\
A_{21} & A_{22}
\end{array}\right], \\
B_{1} & =\left[\begin{array}{ll}
0 & B
\end{array}\right]^{T}, \\
C_{1} & =\left[\begin{array}{ll}
C & 0
\end{array}\right] .
\end{aligned}
$$

Let $p=d / d t$; we have

$$
y(t)=C_{1}\left[p I-A_{1}\right]^{-1} B_{1} u_{1}(t)+C_{1}\left[p I-A_{1}\right]^{-1} f_{2}\left(x_{1}(t)\right) .
$$

Then the representations of input-output are given as

$$
\begin{aligned}
D(p) y(t)= & N(p) u_{1}(t)+N_{f}(p) f_{2}\left(x_{1}(t)\right) \\
= & N_{r} p^{n+l-2} u_{1}(t)+N_{1}(p) u_{1}(t) \\
& +C_{1} p^{n+l-2} \frac{d}{d t} f_{2}\left(x_{1}(t)\right)+N_{f_{1}}(p) f_{2}\left(x_{1}(t)\right),
\end{aligned}
$$

where $N(p) / D(p)=C_{1}\left[p I-A_{1}\right]^{-1} B_{1}$,

$$
\begin{aligned}
& N_{f}(p)=C_{1} \operatorname{adj}\left(p I-A_{1}\right), \\
& N(p)=N_{r} p^{n+l-2}+N_{1}(p), \\
& N_{f}(p)=C_{1} p^{n+l-1}+N_{f_{1}}(p) .
\end{aligned}
$$

Now we have

$$
D(p) y(t)=p^{n+l-2}\left(C \frac{\partial f\left(x_{1}(t)\right)}{\partial u^{T}(t)} B\right) u_{1}(t)
$$

$$
\begin{aligned}
& +N_{1}(p) u_{1}(t)+p^{n+l-2} \\
& \times\left\{C\left(\frac{\partial f\left(x_{1}(t)\right)}{\partial u^{T}(t)}-A_{12}\right)\right. \\
& \times\left(A_{21} x(t)+A_{22} u(t)+g_{1}\left(x_{1}(t)\right)\right) \\
& \left.+C\left(\frac{\partial f\left(x_{1}(t)\right)}{\partial x^{T}(t)}-A_{11}\right) f\left(x_{1}(t)\right)\right\} \\
& +N_{f_{1}}(p) f_{2}\left(x_{1}(t)\right)
\end{aligned}
$$$$
=p^{n+l-2}\left(C \frac{\partial f\left(x_{1}(t)\right)}{\partial u^{T}(t)} B\right) u_{1}(t)+N_{1}(p) u_{1}(t)
$$

$$
\begin{aligned}
+p^{n+l-2}\left\{C\left(\frac{\partial f\left(x_{1}(t)\right)}{\partial u^{T}(t)}-A_{12}\right)\right. \\
\times\left(A_{21} x(t)+A_{22} u(t)+g_{1}\left(x_{1}(t)\right)\right) \\
\left.+C\left(\frac{\partial f\left(x_{1}(t)\right)}{\partial x^{T}(t)}-A_{11}\right) f\left(x_{1}(t)\right)\right\}
\end{aligned}
$$

$$
\begin{aligned}
& +C_{1} \Gamma_{n+l-2} p^{n+l-2} f_{2}\left(x_{1}(t)\right) \\
& +N_{f_{2}}(p) f_{2}\left(x_{1}(t)\right),
\end{aligned}
$$

where $N_{f_{1}}(p)=C_{1} \Gamma_{n+l-2} p^{n+l-2}+N_{f_{2}}(p)$. 
Let

$$
\begin{aligned}
\Gamma_{n+l-1} & =I, \\
\Gamma_{n+l-2} & =A_{1} \Gamma_{n+l-1}+\alpha_{n+l-1} I \\
& =A_{1}+\alpha_{n+l-1} I \\
& =A_{1}-T_{r}\left(A_{1}\right) I,
\end{aligned}
$$

where $\alpha_{n+l-1}=-T_{r}\left(A_{1}\right)$. follows:

Now the representations of input-output (12) are given as

$$
\begin{aligned}
D(p) y(t)= & p^{n+l-2}\left(C \frac{\partial f\left(x_{1}(t)\right)}{\partial u^{T}(t)} B\right) u_{1}(t) \\
& +N_{1}(p) u_{1}(t)+p^{n+l-2} f_{4}\left(x_{1}(t)\right) \\
& +N_{f_{2}}(p) f_{2}\left(x_{1}(t)\right),
\end{aligned}
$$

where

$$
\begin{aligned}
& f_{4}\left(x_{1}(t)\right) \\
& =C\left\{\left(\frac{\partial f\left(x_{1}(t)\right)}{\partial x^{T}(t)}-T_{r}\left(A_{1}\right) I\right) f\left(x_{1}(t)\right)\right. \\
& +\left(\frac{\partial f\left(x_{1}(t)\right)}{\partial u^{T}(t)}\right) g_{1}\left(x_{1}(t)\right) \\
& +\left(\frac{\partial f\left(x_{1}(t)\right)}{\partial u^{T}(t)}-A_{12}\right) \\
& \cdot\left(A_{21} x(t)+A_{22} u(t)\right)+\left(T_{r}\left(A_{1}\right) I-A_{11}\right) \\
& \left.\cdot\left(A_{11} x(t)+A_{12} u(t)\right)\right\} .
\end{aligned}
$$

Let $N_{r} v_{z}(t)=\left(C\left(\partial f\left(x_{1}(t)\right) / \partial u^{T}(t)\right) B\right) u_{1}(t)+f_{4}\left(x_{1}(t)\right)$.

We have

$$
\begin{aligned}
D(p) y(t)= & p^{n+l-2} N_{r} v_{z}(t)+N_{1}(p) u_{1}(t) \\
& +N_{f_{2}}(p) f_{2}\left(x_{1}(t)\right) .
\end{aligned}
$$

The reference model is given as

$$
\begin{gathered}
\dot{x}_{m}(t)=A_{m} x_{m}(t)+B_{m} r_{m}(t) \\
y_{m}(t)=C_{m} x_{m}(t) .
\end{gathered}
$$

Choose a stable polynomial $T(p)$ which satisfies the following conditions. (1) The degree of $T(p)$ is $\rho \geq n_{d}+2 n-$ $n_{m}-2-\eta_{i}$. (2) The coefficient of the maximum degree term of $T(p)$ is the same as $D(p)$.

Consider the following equation:

$$
T(p) D_{m}(p)=D_{d}(p) D(p) R(p)+S(p),
$$

where the degree of each polynomial is $\partial T(p)=\rho, \partial D_{m}(p)=$ $n_{m}, \partial D_{d}(p)=n_{d}, \partial D(p)=n, \partial R(p)=\rho+n_{m}-n_{d}-n$, and $\partial S(p) \leq n_{d}+n-1 . T(p), D_{m}(p), D_{d}(p), D(p)$, and $R(p)$ are monic polynomials.

In this paper, we propose a design of model following control system with disturbances. We can prove that all the internal states are bounded and output error

$$
e(t)=y(t)-y_{m}(t)
$$

converges to zero asymptotically. Then the following form is obtained:

$$
\begin{aligned}
& T(p) D_{m}(p) e(t) \\
& =D_{d}(p) R(p)\left\{p^{n+l-2} N_{r} v_{z}(t)\right. \\
& \left.+N_{1}(p) u_{1}(t)+N_{f_{2}}(p) f_{2}\left(x_{1}(t)\right)\right\} \\
& +S(p) y(t)-T(p) D_{m}(p) y_{m}(t),
\end{aligned}
$$

where $N_{1}(p)=\sum_{k=0}^{n+l-3} C_{1} \Gamma_{k} p^{k} B_{1}=N_{f_{2}}(p) B_{1}$.

The control law (controller) $v_{z}(t)$ can be obtained by making the right-hand side of (22) equal to zero.

Thus,

$$
\begin{aligned}
v_{z}(t)= & -N_{r}^{-1} Q(p)^{-1}\left\{D_{d}(p) R(p) p^{n+l-2}-Q(p)\right\} N_{r} v_{z}(t) \\
& -N_{r}^{-1} Q(p)^{-1} D_{d}(p) R(p) N_{f_{2}}(p) \\
& \cdot\left\{B_{1} u_{1}+f_{2}\left(x_{1}(t)\right)\right\} \\
& -N_{r}^{-1} Q(p)^{-1} S(p) y(t)+r_{m}(t) \\
v_{m}(t)= & N_{r}^{-1} Q(p)^{-1} T(p) N_{m}(p) r_{m}(t) .
\end{aligned}
$$

Therefore, $v_{z}(t)$ of (22) is obtained from $e(t)=0$. The model following control system can be realized if the system internal states are bounded.

\section{Bounded Analysis of Control System Internal State}

Let $z_{1}(t)=\left[\begin{array}{llll}x_{1}^{T}(t) & \xi_{1}^{T}(t) & \xi_{2}^{T}(t) & \xi_{3}^{T}(t)\end{array}\right]^{T}$; then the system is defined by

$$
\begin{gathered}
\dot{z}_{1}(t)=A_{s}+\psi\left(z_{1}(t)\right), \\
x_{1}(t)=C_{s} z_{1}(t),
\end{gathered}
$$


where

$$
\begin{aligned}
& A_{s}=\left[\begin{array}{cccc}
A_{1}-B_{1} E_{2} C_{1} & -B_{1} H_{1} & -B_{1} H_{2} & -B_{1} H_{3} \\
-G_{1} E_{2} C_{1} & F_{1}-G_{1} H_{1} & -G_{1} H_{2} & -G_{1} H_{3} \\
G_{2} C_{1} & 0 & F_{2} & 0 \\
-G_{3} B_{1} E_{2} C_{1} & -G_{3} B_{1} H_{1} & -G_{3} B_{1} H_{2} & F_{3}-G_{3} B_{1} H_{3}
\end{array}\right] \text {, } \\
& C_{s}=\left[\begin{array}{llll}
I & 0 & 0 & 0
\end{array}\right] \text {, } \\
& \psi\left(z_{1}(t)\right)=f_{2}\left(x_{1}(t)\right)-B_{1}\left(C \frac{\partial f\left(x_{1}(t)\right)}{\partial u^{T}(t)} B\right)^{-1} \\
& \cdot\left(W\left(x_{1}(t)\right) v_{z}(t)+f_{4}\left(x_{1}(t)\right)\right) \text {, } \\
& \psi\left(z_{2}(t)\right)=G_{3}\left(f_{2}\left(x_{1}(t)\right)\right. \\
& \left.-B_{1}\left(C \frac{\partial f\left(x_{1}(t)\right)}{\partial u^{T}(t)} B\right)^{-1} f_{4}\left(x_{1}(t)\right)\right) \\
& =G_{3} B_{1}\left(C \frac{\partial f\left(x_{1}(t)\right)}{\partial u^{T}(t)} B\right)^{-1} W\left(x_{1}(t)\right) v_{z}, \\
& C \frac{\partial f\left(x_{1}(t)\right)}{\partial u^{T}(t)} B=N_{r}+W\left(x_{1}(t)\right) .
\end{aligned}
$$

The characteristic polynomial $\left|p I-A_{s}\right|$ can be calculated as follows:

$$
\left|p I-A_{s}\right|=|Q(p)|^{2} V_{s}(p) T(p)^{l} D_{m}(p)^{l}
$$

with stable polynomials of $T(p), D_{m}(p),|Q(p)|$, and $V_{s}(p)$. Therefore, $A_{s}$ is also a stable system matrix.

Now, the system can be rewritten as

$$
\begin{gathered}
\dot{z}_{2}(t)=A_{s} z_{2}(t)+\psi\left(z_{1}(t)\right), \\
x_{1}(t)=C_{s} z_{2}(t) .
\end{gathered}
$$

Consider a quadratic Lyapunov function candidate:

$$
\begin{gathered}
V(t)=\frac{1}{2} z_{2}^{T}(t) P_{s} z_{2}(t), \\
\dot{V}(t)=z_{2}^{T}(t) P_{s}\left(A_{s} z_{2}(t)+\psi\left(z_{1}(t)\right)\right) \\
=-\frac{1}{2} z_{2}^{T}(t) Q_{s} z_{2}(t)+z_{2}^{T}(t) P_{s} \psi\left(z_{1}(t)\right) \\
\leq z_{2}^{T}(t) P_{s} \psi\left(z_{1}(t)\right)<0, \\
P_{s} A_{s}+A_{s}^{T} P_{s}=-Q_{s},
\end{gathered}
$$

where $Q_{s}$ and $P_{s}$ are symmetric positive definite matrices defined by (27). If $A_{s}$ is a stable matrix, we can get a unique $P_{s}$ from (31) when $Q_{s}$ is given. Therefore, $z_{2}(t)$ is bounded.

\section{Simulation Results}

This simulation, which proposes static state feedback AQM controller for the time-delay system, verifies the performance.
TABLE 1: Sender 1-2 node and receiver node parameters.

\begin{tabular}{lc}
\hline Parameter & Value \\
\hline Application & $\mathrm{ftp}$ \\
Transport layer protocol & TCP \\
TCP agent SACK bit & False \\
Packet size & 1448 (byte) \\
Acknowledgment size & 40 (byte) \\
Flow number (TCP session number) & $8(+1)$ (flow) \\
(disturbance)
\end{tabular}

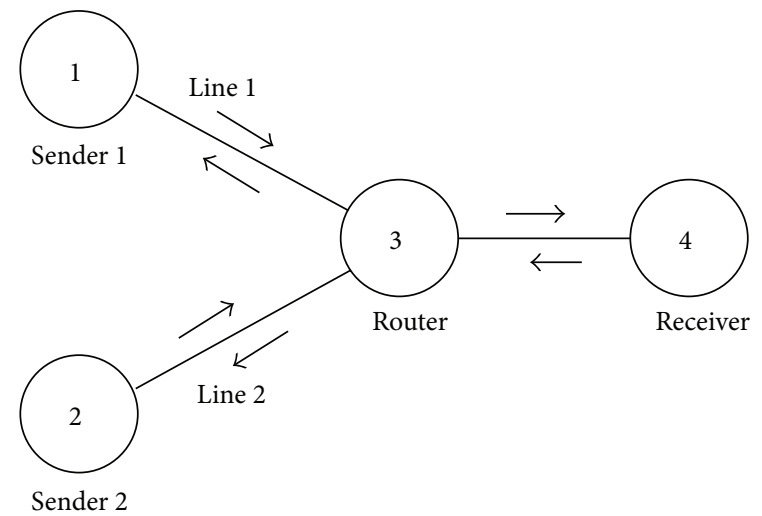

FIgUre 3: The network topology.

The network topology is simple and it is shown in Figure 3. But this small-scale network is developed by using four computers, and some experiments are carried out.

This network consists of two senders with four data flows (this means four sessions and $N=8$ ), one bottleneck router, and one receiver, which receives data from senders through the bottleneck router (see Table 1).

In the responses (Figures 4, 5, and 6) of the congestion system with time-delay, the output errors converge to zero. So the effectiveness of this method is verified.

\section{Conclusions}

In this paper, a new design method for the congestion controller of the TCP/AQM networks is introduced. The developed approach can theoretically guarantee the system performances, including the disturbance rejection and the implied stability of the closed-loop system. This property is useful for congestion controller design. This paper studies a control system with time-delay using a model following method which is one of the effective means of solving time-delay problems in a control system. The method can efficaciously control time-delay under disturbances and has excellent practicability.

By using this model, the nonlinear input time-delay system, which describes a TCP/AQM network, is transformed into an equivalent nonlinear system, and it is possible to design controllers based on nonlinear control theories. For a congestion control problem, a round packet trip time is 


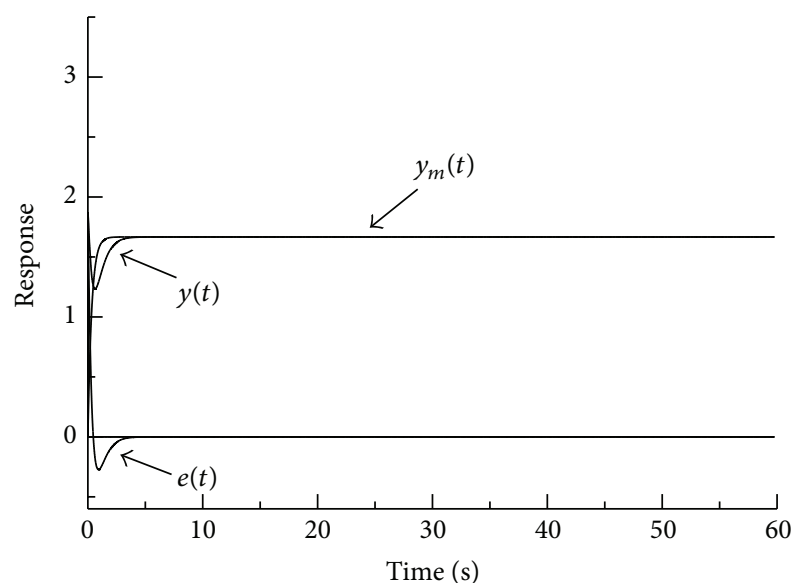

FIGURE 4: Responses of the congestion system with time-delay (the reference queue size is $\bar{q}_{0}=1.7$ (packets); time-delay is $0.12(\mathrm{~ms})$ ).

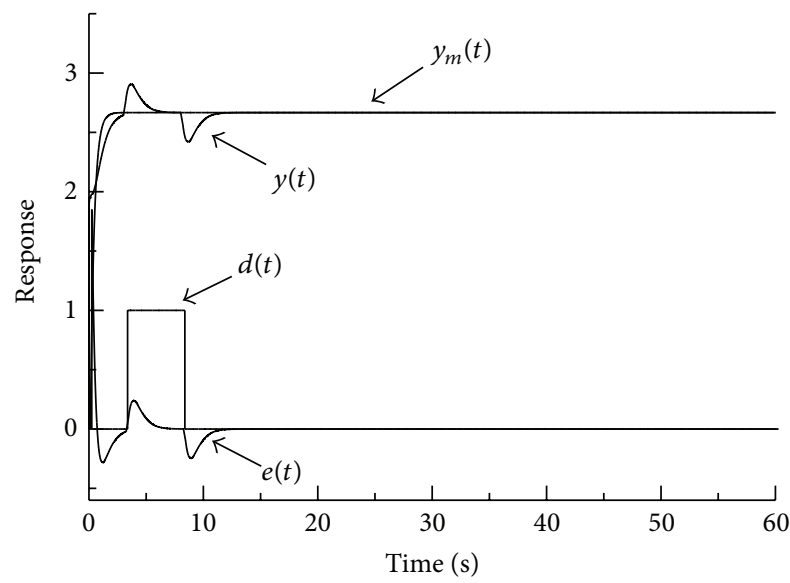

FIGURE 5: Responses of the congestion system with time-delay (the reference queue size is $\bar{q}_{0}=2.7$ (packets), time-delay is 12 (ms), and $d(t)$ is white noises).

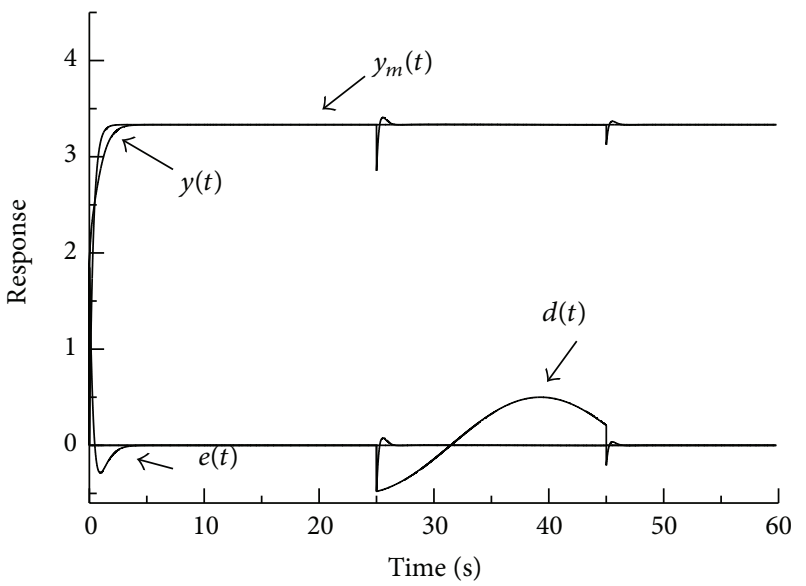

FIGURE 6: Responses of the congestion system with time-delay (the reference queue size is $\bar{q}_{0}=3.3$ (packets), time-delay is 0.1 (ms), and $d(t)$ is white noises). not stationary and depends on the actual traffic. Finally, it is pointed out that the effectiveness of the proposed approach is only verified via simulations.

\section{Conflict of Interests}

The authors declare that there is no conflict of interests regarding the publication of this paper.

\section{Acknowledgments}

This work was financially supported by the Shanghai University of Engineering Science Development Fund to cultivate high-level special project (2012gp02). The authors would like to thank the editor and the reviewers for their constructive comments and suggestions which improved the quality of the paper.

\section{References}

[1] R. Barzamini, M. Shafiee, and A. Dadlani, "Adaptive generalized minimum variance congestion controller for dynamic TCP/AQM networks," Computer Communications, vol. 35, no. 2, pp. 170-178, 2012.

[2] Y. Jing, H. Wang, W. Pan, and X. Liu, "Robust stability analysis for uncertain state and input delay TCP/AQM network systems," in Proceedings of the American Control Conference (ACC '08), pp. 2643-2647, Seattle, Wash, USA, June 2008.

[3] F. P. Kelly, A. K. Maulloo, and D. Tan, "Rate control for communication networks: shadow prices, proportional fairness and stability," Journal of the Operational Research Society, vol. 49, no. 3, pp. 237-252, 1998.

[4] S. H. Low, "A duality model of TCP and queue management algorithms," IEEE/ACM Transactions on Networking, vol. 11, no. 4, pp. 525-536, 2003.

[5] C. V. Hollot, Y. Liu, V. Misra, and D. Towsly, "Unresponsive flows and AQM performance," in Proceedings of the 22nd Annual Joint Conference of the IEEE Computer and Communications (INFOCOM '03), vol. 1, pp. 85-95, March-April 2003.

[6] N. Bigdeli and M. Haeri, "Predictive functional control for active queue management in congested TCP/IP networks," ISA Transactions, vol. 48, no. 1, pp. 107-121, 2009.

[7] S. Manfredi, M. di Bernardo, and F. Garofalo, "Design, validation and experimental testing of a robust AQM control," Control Engineering Practice, vol. 17, no. 3, pp. 394-407, 2009.

[8] F. Zheng and J. Nelson, "An $H_{\infty}$ approach to the controller design of AQM routers supporting TCP flows," Automatica, vol. 45, no. 3, pp. 757-763, 2009.

[9] K. Tsumura, S. Hara, and A. Nakajima, " $\mathrm{H}_{2}$ performance limitation of congestion controller for TCP/AQM network systems," in Proceedings of the 44th IEEE Conference on Decision and Control, and the European Control Conference (CDC-ECC '05), pp. 6768-6773, Seville, Spain, December 2005.

[10] J. Sun, K.-T. Ko, G. Chen, S. Chan, and M. Zukerman, "PD-RED: to improve the performance of RED," IEEE Communications Letters, vol. 7, no. 8, pp. 406-408, 2003.

[11] M. F. Firuzi and M. Haeri, "Adaptive generalized predictive control of active queue management in TCP networks," in Proceedings of the The International Conference on Computer as a Tool (EUROCON 2005), pp. 676-679, Belgrade, Serbia, November 2005. 
[12] L. Tan, W. Zhang, G. Peng, and G. Chen, "Stability of TCP/RED systems in AQM routers," IEEE Transactions on Automatic Control, vol. 51, no. 8, pp. 1393-1398, 2006.

[13] N. Bigdeli and M. Haeri, "CDM-based design and performance evaluation of a robust AQM method for dynamic TCP/AQM networks," Computer Communications, vol. 32, no. 1, pp. 213$229,2009$.

[14] V. Misra, W. B. Gong, and D. Towsley, "Fluid-based analysis of a network of AQM routers supporting TCP flows with an application to RED," in Proceedings of the ACM SIGCOMMConference, pp. 151-160, September 2000.

[15] C. Hollot, V. Misra, D. Towsley, and W. Gong, "On designing improved controllers for AQM routers supporting TCP flows," in Proceedings of 20th Annual Joint Conference of the IEEE Computer and Communications Societies (INFOCOM '01), vol. 3, pp. 1726-1734, IEEE, Anchorage, Alaska, USA, 2001.

[16] S. Floyd and V. Jacobson, "Random early detection gateways for congestion avoidance," IEEE/ACM Transactions on Networking, vol. 1, no. 4, pp. 397-413, 1993.

[17] D. Anick, D. Mitra, and M. M. Sondhi, "Stochastic theory of a data-handling system with multiple sources," The Bell System Technical Journal, vol. 61, no. 8, pp. 1871-1894, 1982.

[18] S. Mascolo, "Congestion control in high-speed communication networks using the Smith principle," Automatica, vol. 35, no. 12, pp. 1921-1935, 1999.

[19] Y. G. Zheng and Z. H. Wang, "Stability and Hopf bifurcation of a class of TCP/AQM networks," Nonlinear Analysis: Real World Applications, vol. 11, no. 3, pp. 1552-1559, 2010.

[20] J. Chiasson and J. Loiseau, Applications of Time Delay Systems, Springer, Berlin, Germany, 2007.

[21] S. Mascolo, "Smith's principle for congestion control in highspeed data networks," IEEE Transactions on Automatic Control, vol. 45, no. 2, pp. 358-364, 2000.

[22] S. Wu, S. Okubo, and D. Wang, "Design of a model following control system for nonlinear descriptor system in discrete time," Kybernetika, vol. 44, no. 4, pp. 546-556, 2008.

[23] D. Wang, S. Wu, and S. Okubo, "The state predictive model following control system for linear time delays," International Journal of Automation and Computing, vol. 6, no. 2, pp. 186-191, 2009.

[24] C. V. Hollot, V. Misra, D. Towsley, and W. Gong, "Analysis and design of controllers for AQM routers supporting TCP flows," IEEE Transactions on Automatic Control, vol. 47, no. 6, pp. 945959, 2002.

[25] C. V. Hollot, V. Misra, D. Towsely, and W. B. Gong, "A control theoretic analysis of RED," in Proceedings of the INFOCOM 12th Annual Joint Conference of the IEEE Computer and Communications Societies, pp. 1510-1519, Anchorage, Alaska, USA, 2001.

[26] B. Marami and M. Haeri, "Implementation of MPC as an AQM controller," Computer Communications, vol. 33, no. 2, pp. 227239, 2010.

[27] T. Azuma, T. Fujita, and M. Fujita, "Congestion control for TCP/AQM networks using state predictive control," EEE Transactions on Electronics, Information and Systems, vol. 125, no. 9, pp. 1491-1496, 2005.

[28] T. Azuma, T. Fujita, and M. Fujita, "A design of state predictive $H_{\infty}$ congestion controllers for TCP/AQM networks," Transactions of the Institute of Systems, Control and Information Engineers, vol. 18, no. 10, pp. 373-375, 2005.

[29] T. Azuma, H. Naito, and M. Fujita, "Experimental verification of stabilizing congestion controllers using the network testbed," in
Proceedings of the American Control Conference, pp. 1841-1846, Portland, Ore, USA, 2005.

[30] Y. Jing, Z. Chen, and G. M. Dimirovski, "Robust fuzzy observerbased control for TCP/AQM network systems with state delay," in Proceedings of the American Control Conference (ACC'10), pp. 1350-1355, Marriott Waterfront, Baltimore, Md, USA, July 2010. 


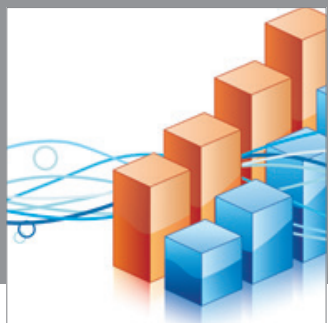

Advances in

Operations Research

mansans

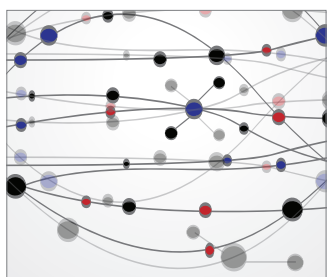

The Scientific World Journal
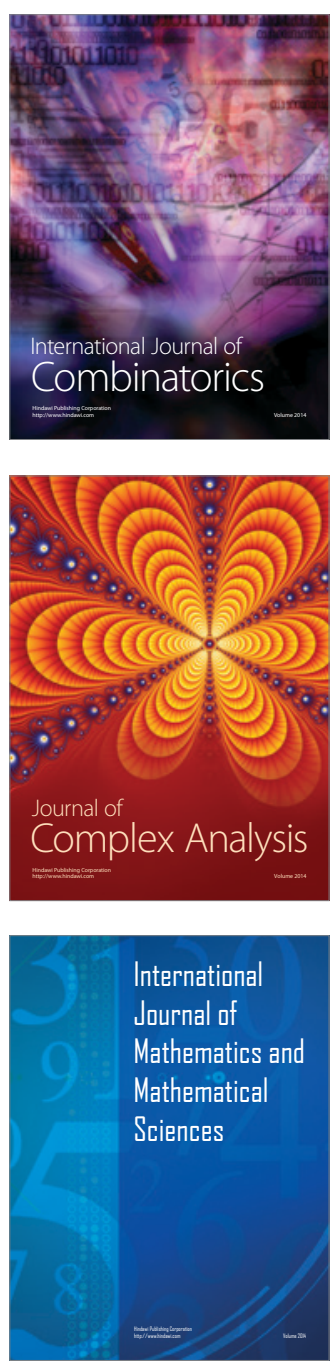
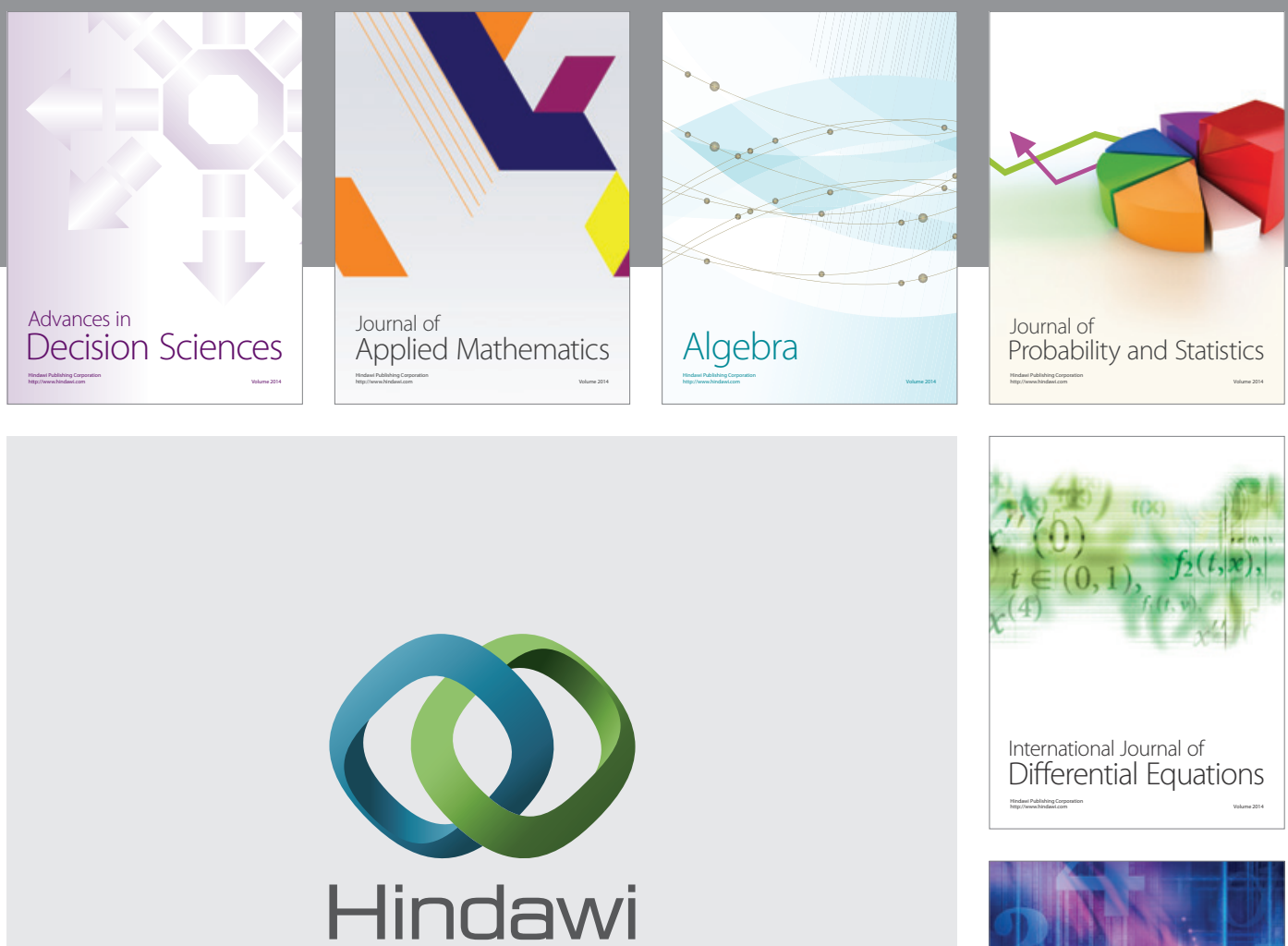

Submit your manuscripts at http://www.hindawi.com
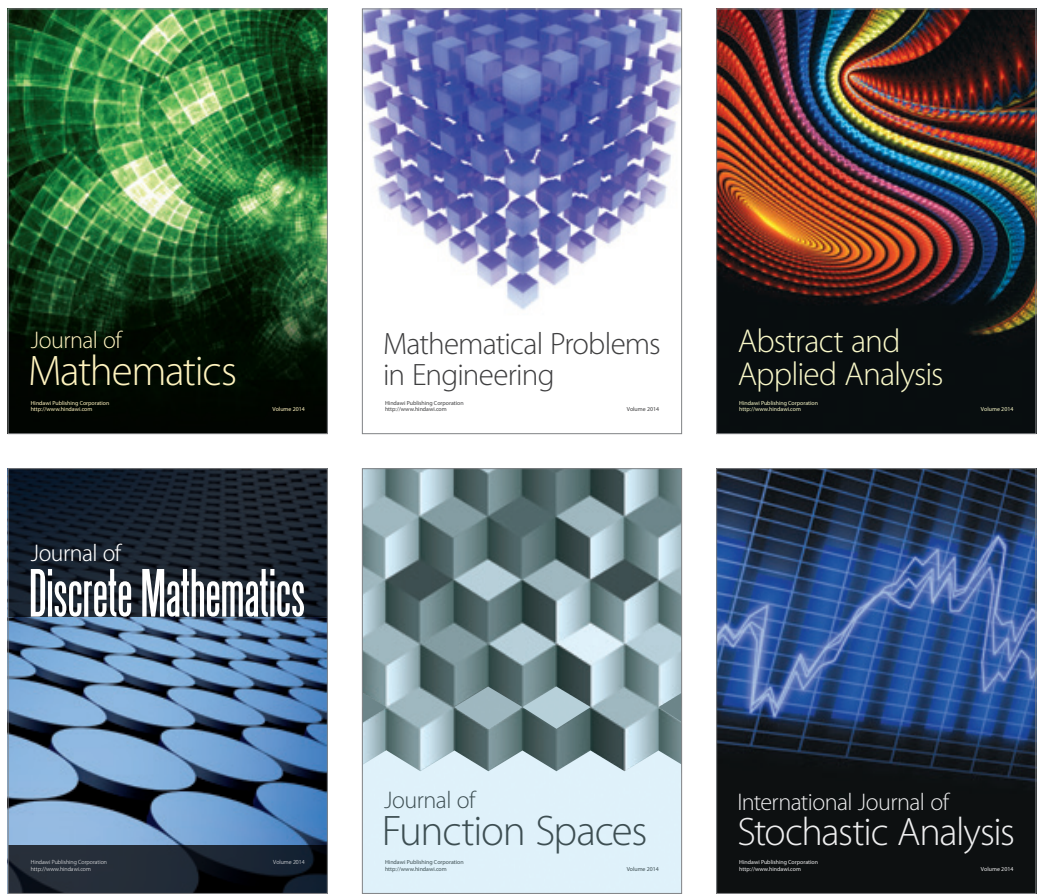

Journal of

Function Spaces

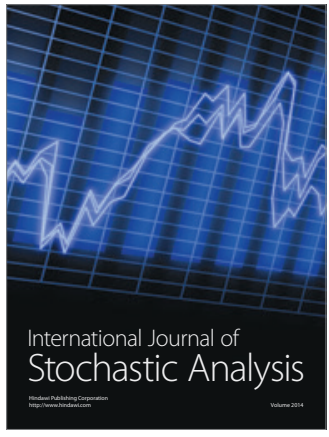

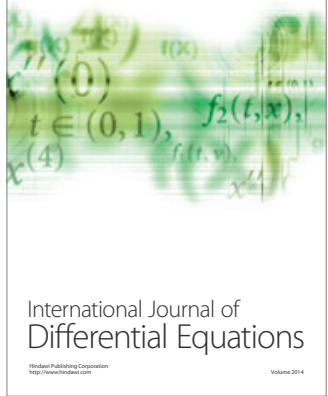
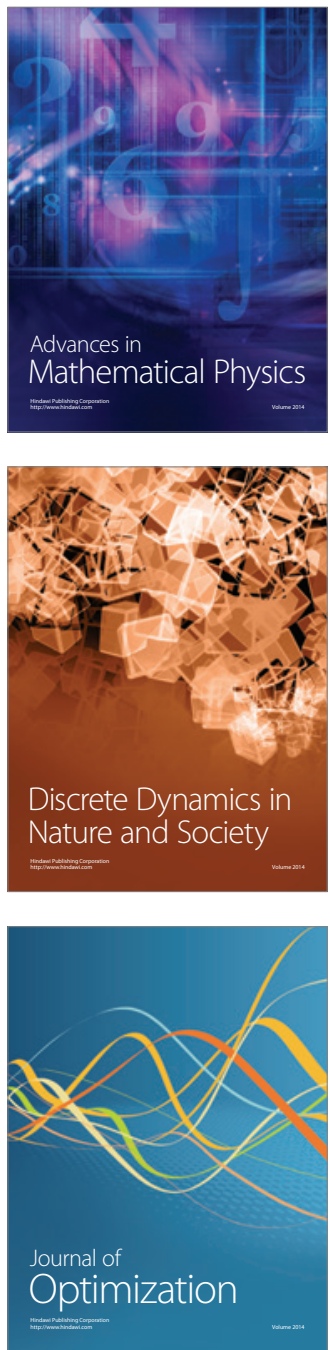